\title{
Following the Path of Microtubules in Bifurcated Dendrites: Using FIB/SEM Microscopy for Ultrastructural Volume Reconstruction of Cortical Tissue
}

\author{
Andreas Schertel*, Yoshiyuki Kubota** \\ * Carl Zeiss NTS GmbH, Carl-Zeiss-Str. 56, D-73447 Oberkochen, Germany \\ ** Division of Cerebral Circuitry, National Institute for Physiological Sciences, \\ Okazaki, Japan; Department of Physiological Sciences, The Graduate University for \\ Advanced Studies (SOKENDAI), Okazaki, Japan; JST, CREST, Tokyo, Japan
}

Recently connectomics, the ultrastructural volume reconstruction of neuronal microcircuits and their synaptic connectivity, has received increased attention in neuroscience. The investigation of the ultrastructural design of neuronal network is very important with regard to brain function. The ultimate goal of connectomics is the complete 3D ultrastructural reconstruction of at least one unit cell of cortical microcircuitry[1].

For electron microscopy, the conventional method is the reconstruction from thin TEM sections obtained by serial slicing of embedded biological tissue by a glass or diamond knife using a microtome[2]. With this preparation technique the z-resolution for 3D reconstruction is limited, because reliable serial slicing requires a thin section thickness of about $40 \mathrm{~nm}$ or thicker. Below about $40 \mathrm{~nm}$ thickness the risk of introducing cutting artefacts and loss of slices escalates. Furthermore only soft materials can be cut by the microtome and the interface between hard materials and the soft biological tissue is not accessible.

For ultrastructural 3D reconstruction of cortical tissue the method of using serial TEM sections is insufficient since many of the structural dimensions like the size of synaptic cleft and vesicles or the diameter of microtubules in dendrites are well below the limited thin section thickness.

The SEM/FIB microscope can provide the solution. In contrast to serial sectioning by microtomy, site specific regions of interest near the surface of biological samples embedded in epoxy can be directly cross-sectioned by FIB milling and imaged for 3D reconstruction. By low $\mathrm{kV}$ surface imaging and by detecting the low loss backscattered electrons from the stained tissue embedded in epoxy, TEM like contrast after inversion of the grey scale and comparable image information is achieved. In a completely automated process it supplies hundreds of serial SEM images of brain tissue in hours. The resolution of the SEM image is sufficient for neuron structure investigation including synapse identification[2,3].

In this study the microtubules in bifurcated dendrites are reconstructed in volume using FIB/SEM microscopy. The analysis is very important with regard to the recently found morphological principle for dendrite dimension; the total cross-sectional area is conserved at dendritic bifurcation points, because the number of microtubule is known to be linearly correlated with cross sectional area of the dendrite[5]. Functionally, this principle is the key factor for allowing neurons to receive a huge number of synapse inputs of almost equal power.

References

[1] J. DeFelipe, Front. Neurosci. 2, 1: 10-12 (2008)

[2] Y. Kubota, S. Hatada, Y. Kawaguchi, Front. Neural Circuits 3:4 (2009)

[3] G. Knott, H. et al. Neurosci. 28, 2959-2964 (2008)

[4] A. Merchán-Pérez et al. Front. Neuroanat. 3:18.(2009)

[5] K.M. Harris et al. in SFN abstract. 147.119 (2007). 
[6] This work was supported by grant-in-aid for scientific research from the Ministry of Education, Culture, Sports, Science and Technology of Japan

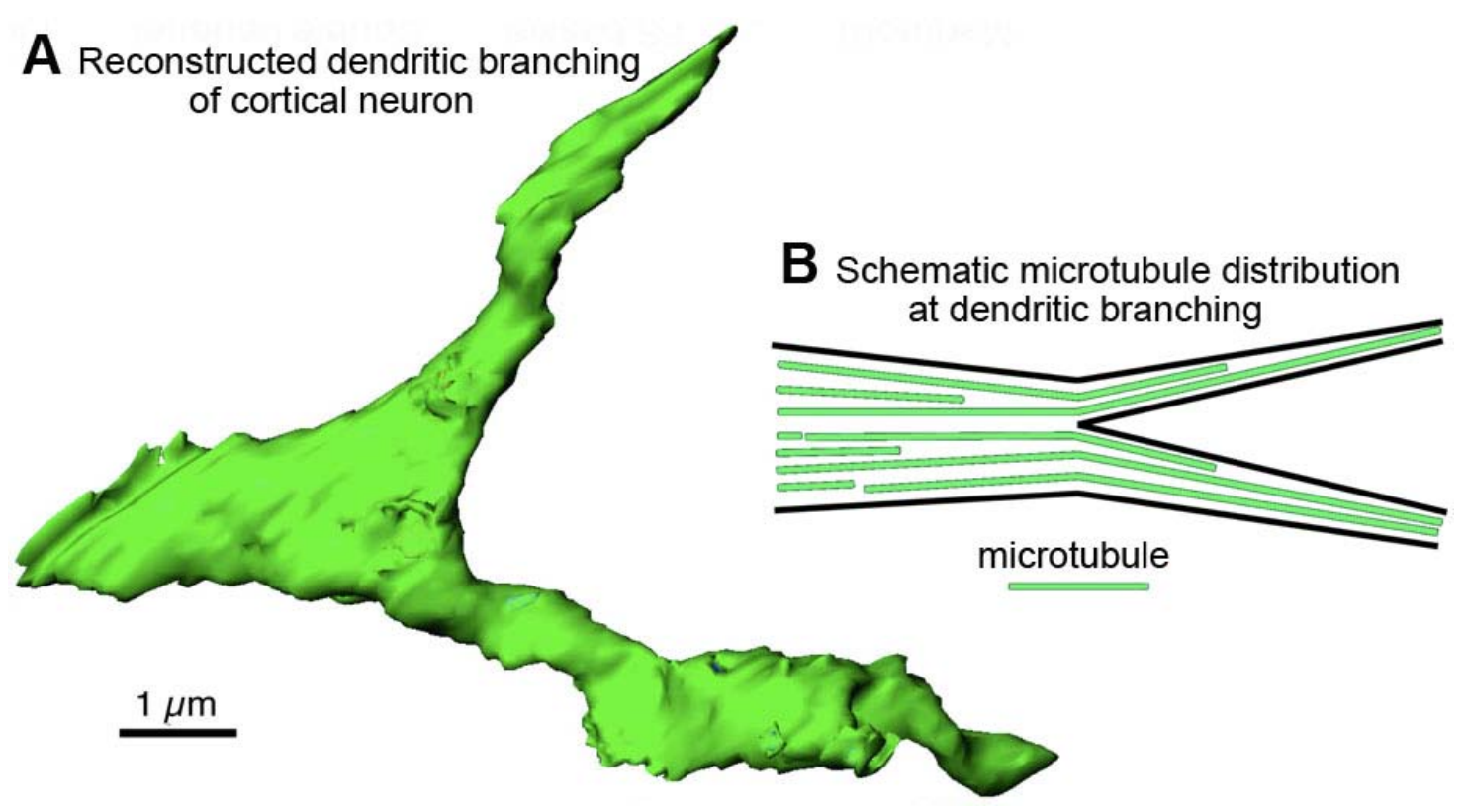

FIG. 1: Microtubule distribution at dendritic branching
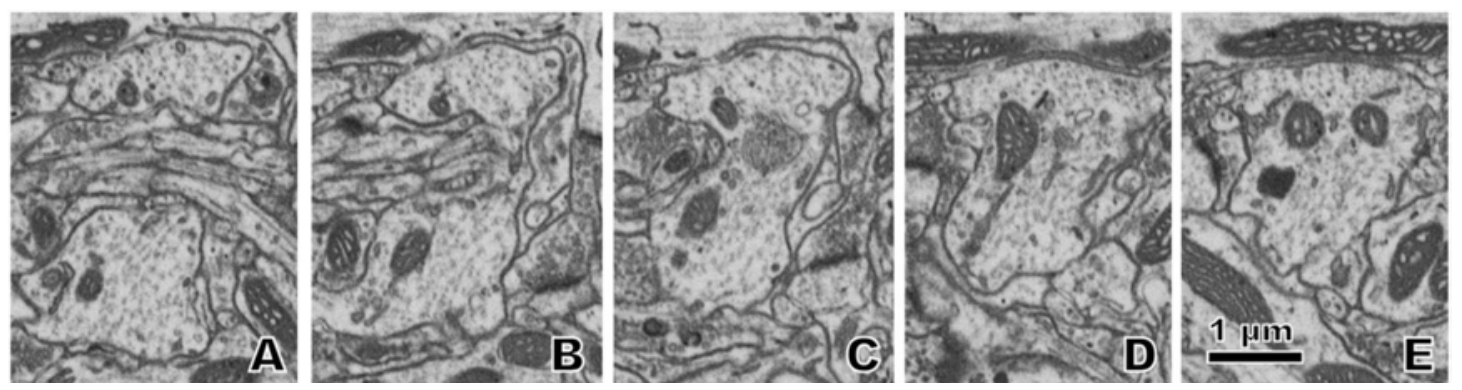

FIG. 2: Representative serial SEM images using FIB slicing of bifurcated dendrite by low $\mathrm{kV}$ back-scattered electron detection 